\title{
DATA FUSION CHALLENGES IN PRECISION BEEKEEPING: A REVIEW
}

\author{
*Nikolajs Bumanis \\ Latvia University of Life Sciences and Technologies, Latvia \\ *Corresponding author’s email: nikolajs.bumanis@gmail.com
}

\begin{abstract}
The objective of precision beekeeping is to minimize resource consumption and maximize productivity of bees. This is achieved by detecting and predicting beehive states by monitoring apiary and beehive related parameters like temperature, weight, humidity, noise, vibrations, air pollution, wind, precipitation, etc. These parameters are collected as a raw input data by use of multiple different sensory devices, and are often imperfect and require creation of correlation between time data series. Currently, most researches focus on monitoring and processing each parameter separately, whereas combination of multiple parameters produces information that is more sophisticated. Raw input data sets that complement one another could be pre-processed by applying data fusion methods to achieve understanding about global research subject. There are multiple data fusion methods and classification models, distinguished by raw input data type or device usage, whereas sensor related data fusion is called sensor fusion. This paper analyses existing data fusion methods and process in order to identify data fusion challenges and correlate them with precision beekeeping objectives. The research was conducted over a period of 5 months, starting from October, 2019 and was based on analysis and synthesis of scientific literature. The conclusion was made that requirement of data fusion appliance in precision beekeeping is determined by a global research objective, whereas input data introduces main challenges of data and sensor fusion, as its attributes correlate with potential result.
\end{abstract}

Key words: data fusion methodology, sensor fusion, sensory monitoring, bee colony states, hiveopolis.

\section{Introduction}

Precision beekeeping $(\mathrm{PB})$ is an apiary management strategy with the focus on monitoring individual bee colonies aimed to minimize resource consumption and maximize the productivity of bees (Zacepins, Stalidzans, \& Meitalovs, 2012). Strategy includes data collection phase, data processing phase and data output phase. Data collection phase corresponds to gathering data about various physical variables associated with bee colonies (Meikle \& Holst, 2015), such as temperature, humidity, respiratory gases, vibration and sound. Data collection is typically performed using sensors that are integrated into bee hives and are connected to the main processing system (Kviesis et al., 2015). Processing phase of bee colony data is typically limited to basic statistical analysis (Henry et al., 2019) with the aim to determine such bee colony states as queenlessness, broodlessness, pre-swarming, swarming and after swarming. Data output phase includes methods to provide processed data - information, to end user in a form of a graphical or tabular representation.

There are multiple studies (Ferrari et al., 2008; Kviesis \& Zacepins, 2015; Meikle \& Holst, 2015; Zacepins et al., 2015) aimed to identify approaches to gather data for analysis, and also define the types of these data. Some of these studies (Human et al., 2013) also propose classification of data collection phase. The limitation of these studies lies in gathering data for particular physical variables like temperature or weight with the aim to analyse this variable by itself. In modern apiaries data if collected through the use of wireless network technologies (Debauche et al., 2018; Henry et al., 2019), can lead to data imperfections and inconsistency. To alleviate such problems, data fusion methods are applied prior to data processing.

There are multiple studies (Castanedo, 2013; Khaleghi et al., 2013; Zheng, 2015; Atluri, Karpatne, \& Kumar, 2018; Beddar-Wiesing \& Bieshaar, 2020) addressing the classification of data fusion methods. However, there is no finalized accepted data fusion classification, and researchers are still proposing new variations.

In the framework of $\mathrm{PB}$, application of data fusion methods can lead to solving global objectives of bee colony lifecycle, such as colony overall health status and colony collapse disorder. However, appliance of data fusion methods in PB is not commonly used practice. The aim of this research was to identify PB oriented data sets and objectives, which can only be achieved by appliance of data fusion methods.

This research was performed in the framework of HIVEOPOLIS project that aims to make technologies available to honeybees that are naturally inaccessible for them (internet, databases, satellite data, robots, etc) and to feed information collected by bees through these channels back to us researchers and also to other hives.

\section{Materials and Methods}

The research was conducted over a period of 5 months, starting from October, 2019, and was based on analysis and synthesis of scientific literature, which addresses data fusion terminology, methodology and application, multi sensor monitoring in beekeeping and development of sensory systems to determine various physical apiary oriented variables and states. The Elsevier's ScienceDirect website was used as the main source for literature acquisition. There are 
currently (on $13^{\text {th }}$ of March) more than 68000 recent (20017-2020) papers on data fusion topic, more than 43000 papers on multi-sensor data, and more than 450 papers on beekeeping related topics.

\section{Results and Discussion}

\section{Data fusion}

Recent fusion terminology proposal (BeddarWiesing \& Bieshaar, 2020) uses data-informationknowledge-wisdom (DIKW) hierarchy as a base to finalize the data fusion oriented terms. In DIKW data is divided (Bellinger, Castro, \& Mills, 2003; Allen, 2004; Targowski, 2005) into five categories: data, information, knowledge, understanding and wisdom. Data is described as representation of objects, whereas processed data leads to information, which can provide answers to 'who', 'what', 'where' and 'when' questions. The application of these data and information generates knowledge, which can provide an answer to 'why' question. If relations and patterns in the information are identified, understanding is reached. As a result, following DIKW hierarchy, I can assume that the quality of data is proportional to the quality of information; therefore, understandability of research object is ensured.

Data fusion as a term is applied to raw data, the first step of DIKW hierarchy. The most accepted definition of data fusion was provided by Joint Directors of Laboratories (JDL) workshop (White, 1991): 'a process dealing with the association, correlation, and combination of data and information from single and multiple sources to achieve refined position and identity estimates, and complete and timely assessments of situations and threats as well as their significance'. Hall and Llinas (Hall \& Llinas, 1997) provided sensor system oriented data fusion definition: 'data fusion techniques combine data from multiple sensors, and related information from associated databases, to achieve improved accuracies and more specific inferences than could be achieved by the use of a single sensor alone'. The employment of terms data and information in these definitions as separate instances can be explained by applying these terms to different data states. The term data fusion is typical in scenarios when data are raw - obtained directly from sensors, whereas the term information fusion is applied to already processed data (Castanedo, 2013). These definitions support my previous assumption about quality data being determinant for quality of information.

According to Castanedo (Castanedo, 2013), data fusion technologies can be classified into three categories, which are data associations, state estimation and decision fusion. This classification was developed based on such criteria as relations between input data sources, input and output data types and their nature, and the abstraction levels of data, which are raw measurements, signals or decisions. The relation between data sources is determined by whether the information was provided by data input sources representing the same or different part of the scene and/or object, whereas the abstraction level corresponds to representation of the input data. In PB data input sources are typically imbedded into beehives; therefore, one set of sensors monitors one particular beehive, rather than an apiary. The Luo et al. (Luo, Yih, \& Su, 2002) proposed such abstraction levels as signal level, pixel level, characteristics level and symbol level.

Alternatively, Zheng (Zheng, 2015) classifies data fusion in three categories, which are featurelevel based, stage based and semantic meaning based. Zheng's stage based data fusion correlates with Castaneda's state estimation. Both of these methods process raw data according to the amount of distinct data sets in sequence. The amount of distinct data sets, or, in perspective of spatial data - layers, leads to quality increase of end result data; however, each of layers may introduce additional fusion challenges. Feature-level based data fusion methods include converting or mapping raw input data into feature vectors that are used for Deep Neural Network (DNN) type data fusion. Liu et al. (Liu et al., 2020) proposed DNN data fusion methods for urban big data with the focus on fusing multi modular data, and Peng et al., (Peng, Deng, \& Chen, 2020) used DNN in combination with Hellinger and Bures metrics for weather data fusion. Both of these studies show that raw input data converted into feature vectors highly increase the scope of possible processing approaches. Both decision fusion and semantic meaning based fusion work with knowledge, rather than raw data; therefore, in this research these methods are not analysed in-depth. Based on DWIK and data fusion classification a hypothesis was put forward: data fusion challenges are mainly raw data related.

To understand the importance of raw data the data fusion process was further analysed. The most basic data fusion process, which is still commonly referenced (Solaiman, 1999; Castanedo, 2013; Zheng, 2015; Chang \& Bai, 2018), was proposed by JDL workshop (White, 1991). JDL has divided data fusion process into five processing levels, which are level 0 - source preprocessing, level 1 - object refinement, level 2 - situation assessment, level 3 impact assessment, and level 4 - process refinement. Source preprocessing includes fusion at the signal and pixel levels. Object refinement - includes process as spatial-temporal alignment, association, correlation, clustering, state estimation, combining of features that were extracted from images. Situation assessment addresses evaluation of relations between 


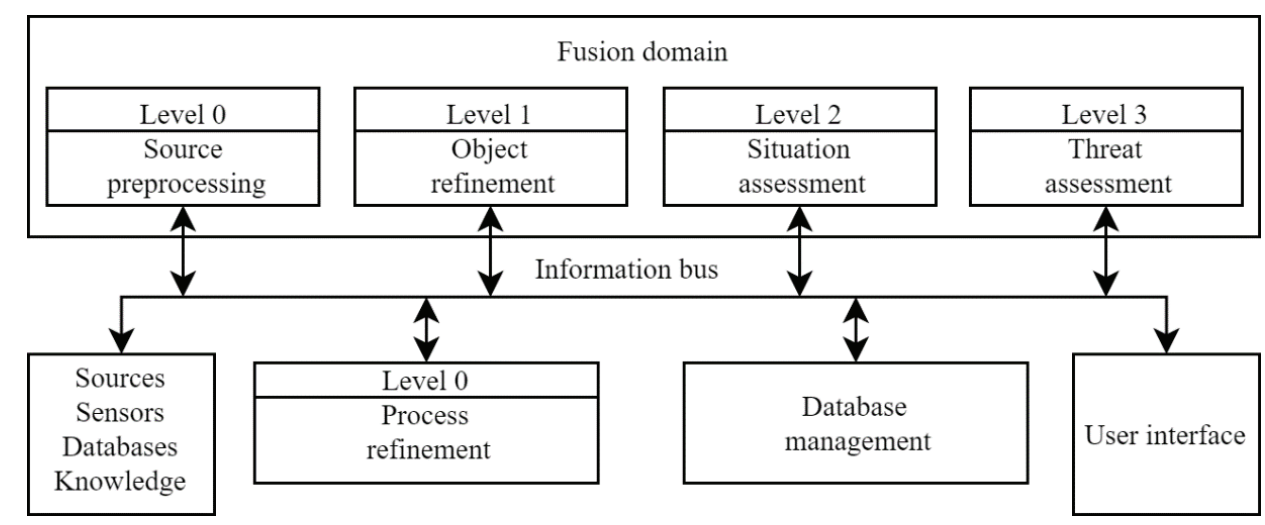

Figure 1. Fusion process according to Joint Directors of Laboratories

(Blasch \& Plano, 2002; Llinas et al., 2004).

object parameters, i.e. proximity, communication, interference, with the aim to identify activities and patterns. Impact assessment evaluates impact of identified activities to obtain a general perspective, i.e. calculates risks, vulnerabilities and operational opportunities. Process refinement uses results from level 0 to level 3 to optimize efficiency of resource management. Based on JDL fusion process definition, the type of raw data is not an obstacle for data fusion, as processing module can fix and adjust it in the second processing step by various means. This leads to taking a previous stated hypothesis as incorrect; however it may change depending on data fusion challenges.

According to Llinas et al. (Llinas et al., 2004) definition of fusion process provided by JDL can only be used as a framework (Figure 1) to understand the functions of data fusion, instead of being taken as a detailed processing architecture.

JDL has many restrictions (Khaleghi et al., 2013) as it is tuned for military applications; however, fusion process can be improved and adjusted, for example, by adding a new level - user refinement (Blasch \& Plano, 2002), which delineates a human from the machine in the process refinement. There can also be improvements to existing levels by addressing the following aspects (Llinas et al., 2004): (1) issues related to quality control, reliability and consistency,
(2) opportunities and needs for co-processing and (3) distributed data fusion. Overall, data fusion method is chosen based on volume and properties of available raw data. Therefore, the data aspect of data fusion process is still determinant to the overall result, and challenges regarding raw data collection and preprocessing were analysed.

Data fusion challenges

In the PB the type of raw data is determined by a sensor or third party source; therefore, the data fusion challenges were first analysed from the perspective of data and its related fusion aspects (Figure 2).

It is acknowledged (Khaleghi et al., 2013) that data provided by sensors are always affected by impreciseness and some degree of uncertainty of the measurements, thus introducing data imperfection. Sensors in PB often become affected by the environment (Kumar, Garg, \& Zachery, 2006; Henry et al., 2019) introducing outliers into raw data sets. It is common in the field of statistics to remove outliers (Zhang, Meratnia, \& Havinga, 2010) prior to performing any analysis and processing.

When using multiple sensors to produce the same physical variable, for example - temperature, conflicting data may be created, thus requiring indepth pre-processing procedures to eliminate such occurrences. In the case of multi sensor systems

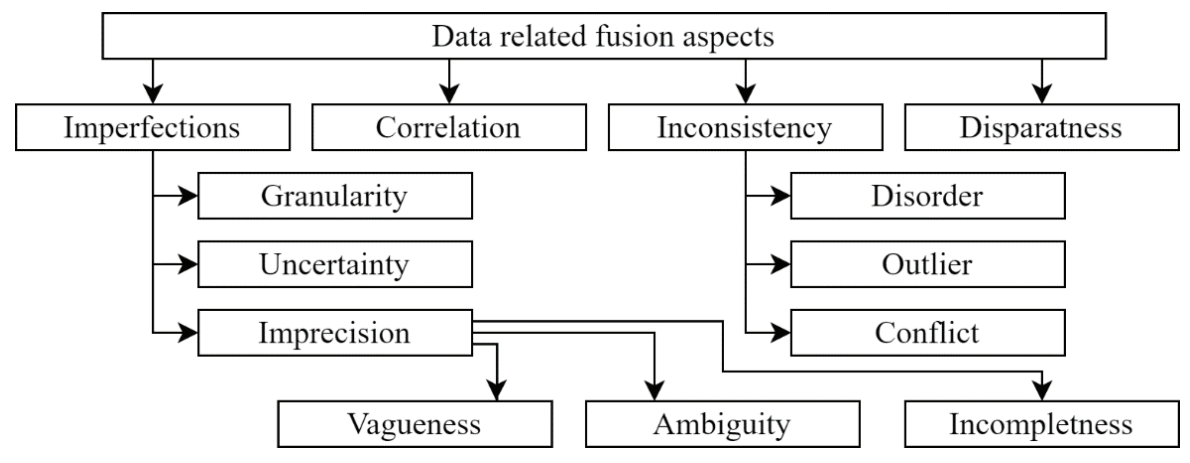

Figure 2. Data related fusion aspects according to Khaleghi et al. (Khaleghi et al., 2013). 
(Kviesis et al., 2015; Henry et al., 2019) that gather data in order to provide decision support, data produced can be multimodal - qualitatively similar (homogenous) or different (heterogeneous), such as auditory, visual, tactile measurements, textual or a mixture data. The heterogeneous data can be divided (Liu et al., 2020) into spatial data, temporal data, static data, dynamic data and attribute data. These representations can also be used in combination with temporal data, i.e. spatial-temporal data (Atluri, Karpatne, \& Kumar, 2018), which contain both time dimension and space dimension. In PB, spatial-temporal data are used for weather forecast.

In case of wireless sensor networks (Meikle \& Holst, 2015; Ampatzidis et al., 2016; Kviesis, Komasilovs, \& Komasilova, 2020) sensor nodes are likely to be exposed to the same external noise that can bias measurements of these sensors; therefore, it is important to establish correct data correlation. This is especially important for large industrial grade apiaries. In addition, acquisition of data from sensors may introduce a problem called data alignment of registration, which occurs when data from each sensor's local frame are transformed into a common frame prior to fusion process (Khaleghi et al., 2013).

Another challenging problem is data association, which occurs in scenarios of multi-target tracking, typically divided into two forms (Sheng et al., 2018): measurement-to-track and track-to-track association. The former refers to the challenge of identifying the source of data, while the latter refers to the problem of distinguishing between tracks.

Either local sensor node or central computer processes the data, thus introducing centralised and decentralised sensor system architecture. Decentralised architecture is preferable in case of wireless sensor networks (Murakami et al., 2007; Kviesis \& Zacepins, 2015; Debauche et al., 2018), as it allows each sensor to process data locally, i.e., eliminating outliers and other imperfections. Appliance of decentralised architecture in PB allows each sensor node to be responsible for particular physical variable's observation. As there are typically (Ferrari et al., 2008; Chang \& Bai, 2018; Debauche et al., 2018) multiple modules per beehive, each for particular variable, decentralised architecture, where each module can process raw data itself, reduces the overall load of main system.

Depending on sensor system architecture, raw input data can be compressed into lower dimensional data, introducing some level of compression loss (Zhu et al., 2005).

Multi sensor data collection also introduces such challenge as operational timing. Data from sensors may be collected in different timeframes, thus requiring for data fusion algorithm to implement varying time scales. The main issue with different timeframes, especially in real-time applications, is the out-of-sequence arrival of data (Besada-Portas et al., 2011). Operational timing can also introduce another challenge - processing the static and dynamic data. The former refers to data that are time-invariant, while latter - to data varying with time. In some cases the latter may require incorporating history of measurements to perform data fusion correctly (Brooks et al., 2009) in order to acquire knowledge about data freshness, i.e. how quickly data sources capture changes and update accordingly (Khaleghi et al., 2013).

Data of precision beekeeping

The type of raw data in PB is determined by the source that produces these data, whereas there are currently multiple variations of data sources in the field of $\mathrm{PB}$.

Primarily the distinction between data levels must be defined. There are three distinct levels defined (Human et al., 2013; Zacepins \& Stalidzans, 2013): apiary, colony and individual bee-related levels.

Apiary level data includes meteorological and video observation data. Main meteorological parameters are wind and precipitations. Apiary management software tend to use (Braga et al., 2020) third party weather stations to acquire these parameters. Spatial observations allow identifying the type of fields and crops (Atluri, Karpatne, \& Kumar, 2018; CalatayudVernich at al., 2019) that are usable for bee foraging. The sources of apiary level data are broad angle video cameras, local apiary weather stations, public weather stations and satellite imagery services.

Colony level data includes temperature, humidity, weight, sound, vibration and video data. Temperature, weight and humidity are the most popular parameters (Stalidzans \& Berzonis, 2013; Meikle \& Holst, 2015), whereas swarming and colony death are the most popular (Ferrari et al., 2008; Kridi, De Carvalho, \& Gomes, 2014) monitor objectives. Researchers use these parameters to determine such beehive states as broodlessness, intensive brood rearing, swarming, pre-swarming and after swarming, overheating, as well as colony death. Sound and video data are also used to determine air and noise pollution. Researchers use sound and vibrations (Bencsik et al., 2015) to determine such beehive states as quenlessness, broodlesness, swarming (including prior and after swarming periods), beehive overpopulation and colony death. The sources of colony level data are temperature sensors, humidity sensors, weight sensors, noise and sound receivers, mono and multispectral video cameras.

The individual bee-related monitoring addresses such objectives as bee counting, i.e., bees going in/out of hive (Souza Cunha et al., 2020), amount of infested 
bees (Bjerge et al., 2019), and bee activity (Ngo et al., 2019). The sources of individual bee-related data are mainly mono and multispectral video cameras.

Depending on the data level and parameters, researchers and apiary management system developers define a system architecture that can realise these principles (Murakami et al., 2007; Kviesis et al., 2015; Kridi, de Carvalho, \& Gomes, 2016; Zacepins et al., 2017a; Zacepins et al., 2017b, Debauche et al., 2018): (1) use as few sensors as possible to minimize diminishing returns, (2) optimize the efficiency of sensor workload by using on-off cycles, (3) use web/ cloud based storage, (4) sufficient scalability for future upgrades.

Data fusion approach applications in precision beekeeping

Based on conducted research, I can conclude that applications of data fusion approach in PB define the requirements for data sets with applications targeting broad objectives, such as spatial positioning of the beehive colony and short-term prediction of weather conditions.

Spatial positioning refers to selection of most efficient position of individual beehive colonies in the framework of available apiary borders. The efficiency is determined by the amount of honey produced by bees during a particular period. The following data sets, respectively, raw data layers for data fusion, must be included into processing: the location, size and borders of an apiary, the types and sizes of nearby fields and available vegetation (including seasonal blooming), the status of pesticide or other harmful chemical use on these fields, nearby and dividing roads, Earth's terrain and its landforms. The amount of bees leaving and entering beehive during a particular period and the changes in the weight of beehive during this period must be taken into account as well. The objective of short-term prediction of weather conditions refers to predicting wind and precipitations in the closest two hours in order to manage bee lifecycle, i.e. automatically closing the beehive gates or changing inside temperature. Henessy et al. proved (Hennessy et al., 2020) that wind has direct and indirect effects on foraging of worker bees (Apis mellifera). The former indicates that foraging rate lowers with increase of wind speed, while latter introduces hesitation of taking off from flowers after nectar gathering. Wind can also transfer harmful substances from nearby fields to foraging areas (Gamboa et al., 2020). Precipitations affect foraging rate and bee lifespan as heavy rain can break bee's wings. He et al. proved (He et al., 2016) that bees work harder before heavy precipitations. The following data sets must be included into processing: air humidity in a particular period, beehive inside/ outside temperature in a particular period, weather forecast, wind speed, wind direction.

\section{Conclusions}

Data fusion is not a novel research topic; however, there are still ongoing debates about proper data fusion terminology, methodology and classification. It can be concluded, that data fusion is and will be a hot topic between researchers in the coming years as it correlates well with developing machine learning topic. It can also be concluded, that data fusion approach applications in the framework of precision beekeeping is a novel idea, and is yet to be researched.

The choice of data fusion method depends on the raw input data sets, as input raw data plays the major role in the data fusion process by determining the correctness and quality of information. The sensors or input data do not determine the need of data fusion for beekeeping related data; rather, it is a global objective, which requires the use of multiple multimodal input data provided by a multi sensor system. Practical applications do not introduce objectively serious technical data fusion challenges as do data type and attributes of input data.

\section{Acknowledgements}

Scientific research, publication and presentation are supported by HIVEOPOLIS project that is funded by the Horizon 2020 FET Programme of the European Union under grant agreement No. 824069.

\section{References}

Allen, G.D. (2004). Hierarchy of Knowledge - from Data to Wisdom. International Journal of Current Research in Multidisciplinary (IJCRM), 2(1), 15-23.

Ampatzidis, Y., Tan, L., Haley, R., \& Whiting, M.D. (2016). Cloud-based harvest management information system for hand-harvested specialty crops. Computers and Electronics in Agriculture, 122, 161-167. DOI: 10.1016/j.compag.2016.01.032.

Atluri, G., Karpatne, A., \& Kumar, V. (2018). Spatio-temporal data mining: A survey of problems and methods. ACM Computing Surveys, 51(4), 1-37. DOI: 10.1145/3161602.

Beddar-Wiesing, S., \& Bieshaar, M. (2020). Multi-Sensor Data and Knowledge Fusion - A Proposal for a Terminology Definition. ArXiv. Retrieved January 27, 2020, from http://arxiv.org/abs/2001.04171.

Bellinger, G., Castro, D., \& Mills, A. (2003). Data, Information, Knowledge, and Wisdom. 5-7. Retrieved January 27, 2020, from http://outsights.com/systems/dikw/dikw.htm. 
Bencsik, M., Le Conte, Y., Reyes, M., Pioz, M., Whittaker, D., Crauser, D., ... Newton, M.I. (2015). Honeybee colony vibrational measurements to highlight the brood cycle. PLOS ONE 10(11): No. 1371. DOI: 10.1371/ journal.pone.0141926.

Besada-Portas, E., Lopez-Orozco, J.A., Besada, J., \& De La Cruz, J.M. (2011). Multisensor fusion for linear control systems with asynchronous, Out-Of-Sequence and erroneous data. Automatica, 47(7), 1399-1408. DOI: 10.1016/j.automatica.2011.02.030.

Bjerge, K., Frigaard, C.E., Høgh Mikkelsen, P., Holm Nielsen, T., \& Misbih, M. (2019). A computer vision system to monitor the infestation level of Varroa destructor in a honeybee colony. Computers and Electronics in Agriculture, 164. DOI: 10.1016/j.compag.2019.104898.

Blasch, E.P., \& Plano, S. (2002). JDL level 5 fusion model: user refinement issues and applications in group tracking. In AeroSense 2002, 2002, Orlando, FL, United States. 31 July 2002. Proc. SPIE 4729, Signal Processing, Sensor Fusion, and Target Recognition XI, 4729, 270-279. DOI: 10.1117/12.477612.

Brooks, A., Makarenko, A., Kaupp, T., Durrant-Whyte, H., \& Dellaert, F. (2009). Decentralised Data Fusion with Dynamic Topologies - A Graphical Model Approach. IFAC Proceedings Vol. 42(20), 222-227. DOI: 10.3182/20090924-3-it-4005.00038.

Calatayud-Vernich, P., Calatayud, F., Simó, E., Pascual Aguilar, J.A., \& Picó, Y. (2019). A two-year monitoring of pesticide hazard in-hive: High honey bee mortality rates during insecticide poisoning episodes in apiaries located near agricultural settings. Chemosphere, 232, 471-480. DOI: 10.1016/j.chemosphere.2019.05.170.

Castanedo, F. (2013). A review of data fusion techniques. The Scientific World Journal, 2013, No. 704504, p. 19. DOI: $10.1155 / 2013 / 704504$.

Chang, N., \& Bai, K. (2018). Concepts and Basics of Image and Data Fusion. Multisensor data fusion and machine learning for environmental remote sensing (pp. 168-170). Taylor and Franci Group. Boca Raton, Florida. DOI: 10.1201/b20703.

Gamboa, L.G., Diaz, K.S., Ruepert, C., \& van Wendel de Joode, B. (2020). Passive monitoring techniques to evaluate environmental pesticide exposure: Results from the Infant's Environmental Health study (ISA). Environmental Research, 184(May 2020), No. 109243. DOI: 10.1016/j.envres.2020.109243.

Debauche, O., Moulat, M.El., Mahmoudi, S., Boukraa, S., Manneback, P., \& Lebeau, F. (2018). Web Monitoring of Bee Health for Researchers and Beekeepers Based on the Internet of Things. Procedia Computer Science, 130, 991-998. DOI: 10.1016/j.procs.2018.04.103.

Ferrari, S., Silva, M., Guarino, M., \& Berckmans, D. (2008). Monitoring of swarming sounds in bee hives for early detection of the swarming period. Computers and Electronics in Agriculture, 64(1), 72-77. 10.1016/j. compag.2008.05.010.

Hall, D.L., \& Llinas, J. (1997). An introduction to multisensor data fusion. Proceedings of the IEEE, 85(1), 6-23. DOI: $10.1109 / 5.554205$.

He, X.J., Tian, L.Q., Wu, X.B., \& Zeng, Z.J. (2016). RFID monitoring indicates honeybees work harder before a rainy day. Insect Science, Vol. 23, 157-159. DOI: 10.1111/1744-7917.12298.

Hennessy, G., Harris, C., Eaton, C., Wright, P., Jackson, E., Goulson, D., \& Ratnieks, F.F.L.W. (2020). Gone with the wind: effects of wind on honey bee visit rate and foraging behaviour. Animal Behaviour, 161, 23-31. DOI: 10.1016/j.anbehav.2019.12.018.

Henry, E., Adamchuk, V., Stanhope, T., Buddle, C., \& Rindlaub, N. (2019). Precision apiculture: Development of a wireless sensor network for honeybee hives. Computers and Electronics in Agriculture, 156(June 2018), 138-144. DOI: 10.1016/j.compag.2018.11.001.

Human, H., Brodschneider, R., Dietemann, V., Dively, G., Ellis, J. D., Forsgren, E., ... Zheng, H.Q. (2013). Miscellaneous standard methods for Apis mellifera research. Journal of Apicultural Research, 52(4), DOI: 10.3896/IBRA.1.52.4.10.

Khaleghi, B., Khamis, A., Karray, F.O., \& Razavi, S.N. (2013). Multisensor data fusion: A review of the stateof-the-art. Information Fusion, 14(1), 28-44. DOI: 10.1016/j.inffus.2011.08.001.

Kridi, D.S., de Carvalho, C.G.N., \& Gomes, D.G. (2016). Application of wireless sensor networks for beehive monitoring and in-hive thermal patterns detection. Computers and Electronics in Agriculture, 127, 221235. DOI: 10.1016/j.compag.2016.05.013.

Kridi, D.S., de Carvalho, C.G.N., \& Gomes, D.G. (2014). A predictive algorithm for mitigate swarming bees through proactive monitoring via wireless sensor networks. In Proceedings of the 11th ACM Symposium on Performance Evaluation of Wireless Ad Hoc, Sensor, and Ubiquitous Networks. Association for Computing Machinery, New York, USA, 21-26 September 2014 (pp. 41-47). DOI: $10.1145 / 2653481.2653482$. 
Kumar, M., Garg, D.P., \& Zachery, R.A. (2006). A generalized approach for inconsistency detection in data fusion from multiple sensors. In Proceedings of the American Control Conference, 14-16 June 2006 (pp. 2078-2083). DOI: 10.1109/acc.2006.1656526.

Kviesis, A., Komasilovs, V., \& Komasilova, O. (2020). Application of fuzzy logic for honey bee colony state detection based on temperature data. Biosystems Engineering, 193, 90-100. DOI: 10.1016/j. biosystemseng.2020.02.010.

Kviesis, A., \& Zacepins, A. (2015). System architectures for real-time bee colony temperature monitoring. Procedia Computer Science, 43(C), 86-94. DOI: 10.1016/j.procs.2014.12.012.

Kviesis, A., Zacepins, A., Durgun, M., \& Tekin, S. (2015). Application of wireless sensor networks in precision apiculture. In Proceedings of 14th International Scientific Conference Engineering for Rural Development, 20-22 May 2014 (pp. 440-445). Jelgava, Latvia.

Liu, J., Li, T., Xie, P., Du, S., Teng, F., \& Yang, X. (2020). Urban big data fusion based on deep learning: An overview. Information Fusion, 53(June 2019), 123-133. DOI: 10.1016/j.inffus.2019.06.016.

Llinas, J., Bowman, C., Rogova, G., Steinberg, A., Waltz, E., \& White, F. (2004). Revisiting the JDL data fusion model II. In Proceedings of the Seventh International Conference on Information Fusion, 28 June 1 July 2004 (pp. 1218-1230). Stockholm, Sweden: Computer Science.

Luo, R.C., Yih, C.C., \& Su, K.L. (2002). Multisensor fusion and integration: Approaches, applications, and future research directions. IEEE Sensors Journal, 2(2), 107-119. DOI: 10.1109/JSEN.2002.1000251.

Meikle, W.G., \& Holst, N. (2015). Application of continuous monitoring of honey bee colonies. Apidologie, 46(1), 10-22. DOI: 10.1007/s13592-014-0298-x.

Murakami, E., Saraiva, A.M., Ribeiro, L.C.M., Cugnasca, C.E., Hirakawa, A.R., \& Correa, P.L.P. (2007). An infrastructure for the development of distributed service-oriented information systems for precision agriculture. Computers and Electronics in Agriculture, 58(1), 37-48. DOI: 10.1016/j.compag.2006.12.010.

Ngo, T.N., Wu, K.C., Yang, E.C., \& Lin, T.T. (2019). A real-time imaging system for multiple honey bee tracking and activity monitoring. Computers and Electronics in Agriculture, 163(1), No. 104841. DOI: 10.1016/j.compag.2019.05.050.

Peng, W., Deng, H., \& Chen, A. (2020). Using Hellinger and Bures metrics to construct two-dimensional quantum metric space for weather data fusion. Information Fusion, 55(September 2019), 199-206. DOI: 10.1016/j.inffus.2019.09.004.

Braga, A.R., Gomes, D.G., Rogers, R., Hassler, E., Freitas, B.M., \& Cazier, J.A. (2020). A method for mining combined data from in-hive sensors, weather and apiary inspections to forecast the health status of honey bee colonies. Computers and Electronics in Agriculture, 169(September 2019), No. 105161. DOI: 10.1016/j.compag.2019.105161.

Sheng, X., Chen, Y., Guo, L., Yin, J., \& Han, X. (2018). Multitarget tracking algorithm using multiple GMPHD filter data fusion for sonar networks. Sensors (Switzerland), 18(10), No. 3193. DOI: 10.3390/s18103193.

Solaiman, B. (1999). Multisensor data fusion using fuzzy concepts: application to land-cover classification using ers-1/jers-1 sar composites. IEEE Transactions on Geoscience and Remote Sensing, 37(3 I), 13161326. DOI: $10.1109 / 36.763295$.

Souza Cunha, A.E., Rose, J., Prior, J., Aumann, H.M., Emanetoglu, N.W., \& Drummond, F.A. (2020). A novel non-invasive radar to monitor honey bee colony health. Computers and Electronics in Agriculture, 170, No. 105241. DOI: 10.1016/j.compag.2020.105241.

Stalidzans, E., \& Berzonis, A. (2013). Temperature changes above the upper hive body reveal the annual development periods of honey bee colonies. Computers and Electronics in Agriculture, 90, 1-6. DOI: 10.1016/j. compag.2012.10.003.

Targowski, A. (2005). From Data to Wisdom. Dialogue and Universalism, 15(5), 55-71. DOI: 10.5840/ du2005155/629.

White, F.E. (1991). Data Fusion Lexicon. The Data Fusion Subpanel of the Joint Directors of Laboratories, Technical Panel for C3, USA: U.S. Department of Defense, p. 15. Retrieved March 10, 2020, from https://apps. dtic.mil/docs/citations/ADA529661.

Zacepins, A., Stalidzans, E., \& Meitalovs, J. (2012). Application of Information Technologies in Precision Apiculture. In Proceedings of the 13th International Conference on Precision Agriculture (ICPA 2012), 31 July 3 August 2012, Indianapolis, Indiana USA.

Zacepins, A., Pecka, A., Osadcuks, V., Kviesis, A., \& Engel, S. (2017a). Solution for automated bee colony weight monitoring. Agronomy Research, 15(2), 585-593.

Zacepins, A., Brusbardis, V., Meitalovs, J., \& Stalidzans, E. (2015). Challenges in the development of Precision Beekeeping. Biosystems Engineering, 130, 60-71. DOI: 10.1016/j.biosystemseng.2014.12.001. 
Zacepins, A., Kviesis, A., Pecka, A., \& Osadcuks, V. (2017b). Development of Internet of Things concept for Precision Beekeeping. In Proceedings of $18^{\text {th }}$ International Carpathian Control Conference, 28-31 May, 2017 (p. 23-27). DOI: 10.1109/CarpathianCC.2017.7970365.

Zacepins, A., \& Stalidzans, E. (2013). Information processing for remote recognition of the state of bee colonies and apiaries in precision beekeeping (apiculture). Biosystems and Information Technology, 2(1), 6-10. DOI: $10.11592 /$ bit.130502.

Zhang, Y., Meratnia, N., \& Havinga, P. (2010). Outlier detection techniques for wireless sensor networks: A survey. IEEE Communications Surveys and Tutorials, 12(2), 159-170. DOI: 10.1109/SURV.2010.021510.00088.

Zheng, Y. (2015). Methodologies for Cross-Domain Data Fusion: An Overview. IEEE Transactions on Big Data, 1(1), 16-34. DOI: 10.1109/tbdata.2015.2465959.

Zhu, Y., Song, E., Zhou, J., \& You, Z. (2005). Optimal dimensionality reduction of sensor data in multisensor estimation fusion. IEEE Transactions on Signal Processing, 53(5), 1631-1639. DOI: 10.1109/TSP.2005.845429. 